\title{
Surgical clavicle reconstruction after aneurysmal bone cyst resection in a child: A simple method
}

\author{
Luis Rodríguez-Nogué, $\mathrm{MD}^{1}{ }^{(1)}$, Alberto Hernández-Fernández, $\mathrm{MD}^{1}{ }^{(\mathbb{D})}$, Cristian Pinilla-Gracia, MD, $\mathrm{PhD}^{1}{ }^{(1)}$, \\ Victoria E Gómez-Palacio, MD, $\mathrm{PhD}^{1}$ (D), Jorge Gil-Albarova, $\mathrm{MD}^{1,2}$ (i)
}

${ }^{1}$ Orthopedic and Trauma Surgery Service, Miguel Servet University Hospital, Zaragoza, Spain

${ }^{2}$ Department of Surgery, Faculty of Medicine, University of Zaragoza, Zaragoza, Spain

Bone tumors of the clavicle are exceedingly rare with a reported frequency of less than $1 \%$ of all bone tumors. ${ }^{[1]}$ Because of this, most doctors have limited experience in the diagnosis and treatment of these tumors. The prevalence of aneurysmal bone cyst $(\mathrm{ABC})$ is greater in the first two decades of life, due to its relationship with the immature and growing skeleton, affecting almost twice as many males as females. ${ }^{[2]}$ It is often found in long bones, particularly in the metaphyseal area of the femur and tibia. It can also be located in the spine and pelvis, ${ }^{[3]}$ but its location in the clavicle is very rare. ${ }^{[4]}$ Differential diagnosis should be considered with essential bone cyst, giant cell tumor, osteoblastoma, chondromyxoid fibroma, and telangiectatic osteosarcoma. ${ }^{[4,5]}$ In this article, we propose a description of a simple technique, some considerations about treatment options in selected $\mathrm{ABC}$ located in clavicle during childhood, and a follow-up of more than two years. ${ }^{[6]}$

\section{CASE REPORT}

A seven-year-old boy was referred to our clinic due to a painful tumor of progressive growth, in the lateral-

Received: February 17, 2020

Accepted: March 18, 2020

Published online: April 11, 2020

Correspondence: Luis Rodríguez-Nogué, MD. Orthopedic and Trauma Surgery Service, Miguel Servet University Hospital, 50009, Zaragoza, Spain.

E-mail: luisrodn7@gmail.com

Doi: 10.5606/ehc. 2020.74184

Citation: Rodríguez-Nogué L, Hernández-Fernández A, PinillaGracia C, Gómez-Palacio VE, Gil-Albarova J. Surgical clavicle reconstruction after aneurysmal bone cyst resection in a child: A simple method. Jt Dis Relat Surg 2020;31(2):367-371.

\section{ABSTRACT}

The clavicle is an infrequent location for primary tumors in general, and aneurysmal bone cyst (ABC) of the clavicle is particularly rare. The challenge of the functional and esthetic result in the treatment of these lesions in the pediatric population is high when considering the reconstruction of critical bone defects. In this article, we present the case of a seven-year-old boy with an $\mathrm{ABC}$ in the middle third of the clavicle, treated by resection and reconstruction with free autograft of the fibula stabilized by using an intramedullary titanium nail. We offer a description of the used technique, considerations about treatment options in children, and a follow-up of more than two-and-a-half years.

Keywords: Aneurysmal bone cyst, child, clavicle, free fibular graft, reconstruction method.

middle third of the right clavicle with functional impotence of the limb. The parents reported that the child had been treated for a fracture of the right clavicle five months earlier by a sling in another clinic, although they did not have a previous image study. The X-ray showed a radiolucent diaphyseal lesion, insufflated, centered, well delimited, which thinned the cortex and respected the growth plates and the epiphysis. Through computed tomography and magnetic resonance imaging, a cavitated, multiloculated lesion was observed, with fluid levels inside and with a mild inflammatory reaction of adjacent soft tissues (Figure 1). The scintigraphy study was compatible with the diagnosis of cystic bone injury and bone remodelling in relation to the previous fracture. A diagnosis of pathological fracture on active $A B C$ was then suspected. A written informed consent was obtained from the legal guardian of the patient.

Given the absence of aggressive or malignant cystic lesion behavior in all previous imaging studies, a block resection without prior puncture 

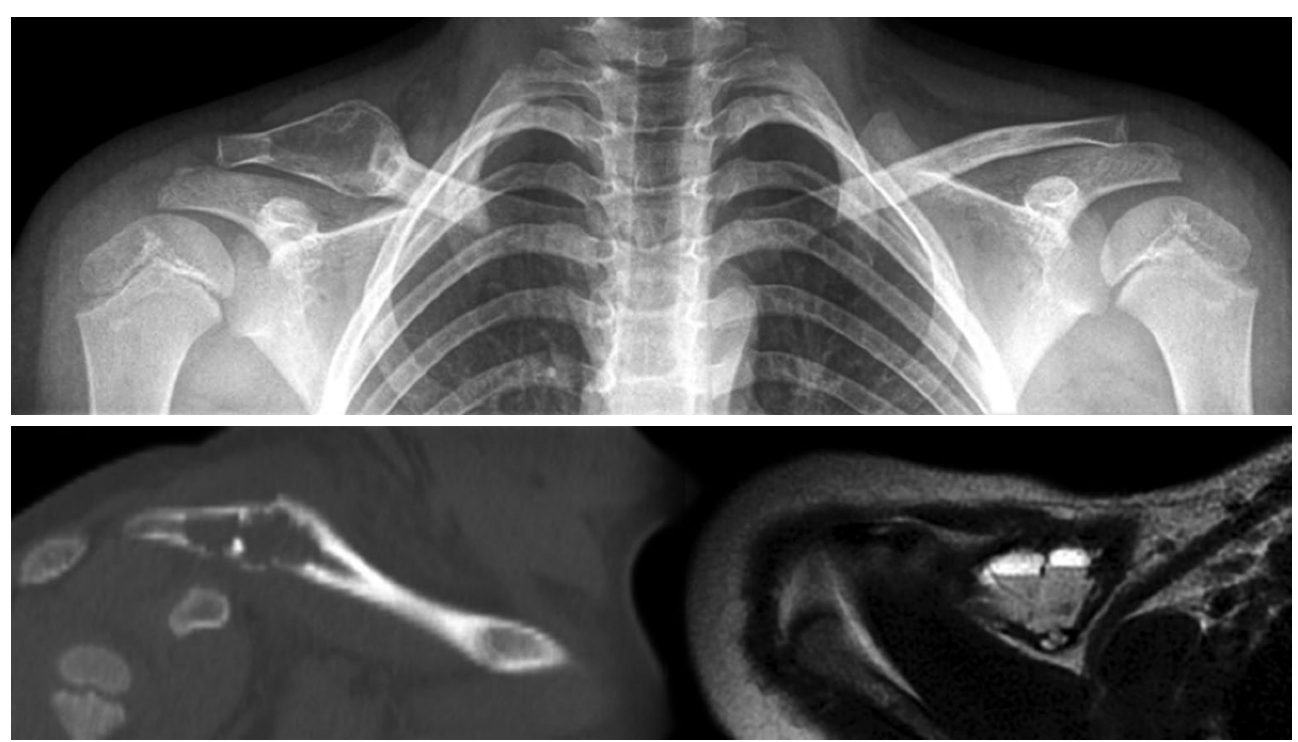

FIGURE 1. Preoperative X-ray, computed tomography and magnetic resonance imaging appearance.

biopsy was chosen due to the existence of an active process of consolidation and remodelling of the pathological fracture suffered, which could condition histopathological interpretation. The surgical treatment consisted of the complete resection of the cyst, preserving the epiphyseal ends. After the resection, the bone surfaces and the resection bed were impregnated with a gauze soaked in a lauromacrogol solution (Etoxisclerol ${ }^{\circledR}$ 2\%) for 10 minutes to reduce the risk of local recurrence since the cyst showed some perforation in its bone boundaries. The reconstruction of the resulting critical bone defect $(4.5 \mathrm{~cm}$ in length) was performed by free autologous bone graft taken subperiosteally from the diaphysis of the ipsilateral fibula (Figure 2). The length of the obtained graft was $3 \mathrm{~mm}$ greater than the length of the bone defect to ensure its compression fit. Its stabilization was achieved by means of a $2 \mathrm{~mm}$ intramedullary titanium nail (TEN, Synthes ${ }^{\circledR}, \mathrm{GmbH}$, Oberdorf, Switzerland) introduced in a retrograde-anterograde manner, leaving the lateral end subcutaneous (Figure 3).

After careful suture of the periosteal cuff of the donor area, the leg was immobilized for three weeks by using a short posterior splint, avoiding weight bearing. In the postoperative period, the right shoulder was kept in a sling for four weeks, progressively allowing the active mobilization of the limb, avoiding risky sports for six months. Histopathological analysis confirmed the diagnosis of $\mathrm{ABC}$, with free resection margins (Figure 4).

The clinical and radiographic evolution was satisfactory (Figure 5), evidencing a satisfactory radiographic incorporation and consolidation of the graft at five months postoperatively. The joint balance of the shoulder was completely restored six weeks after the intervention. The extraction of the intramedullary nail was performed under sedation 14 months after the intervention, without incidents. After 18 months of the intervention, the child presented complete mobility of the shoulder, without pain or deformities, and the complete reconstruction of the donor area of the graft could be verified.

Currently, after more than two-and-a-half years of follow-up, the child maintains an optimal functional outcome, without complications or sequels at the level of the clavicle or in the donor area of the fibula, and without signs of recurrence of the lesion.

\section{DISCUSSION}

Clavicular location of the $\mathrm{ABC}$ is very uncommon, with a limited number of published cases. ${ }^{[1,4]}$ There are different therapeutic options for the treatment, such as curettage with or without grafting, resection with or without reconstruction, arterial embolization, and even radiotherapy. ${ }^{[5,7-10]}$

Yashavntha et al. ${ }^{[8]}$ published the case of a sevenyear-old girl with a clavicle ABC treated by curettage and filling the cavity with a mixture of calcium sulfate and bone allograft, without complications or recurrence of the lesion after one year of follow-up.

Smith ${ }^{[9]}$ collected two cases of clavicle $A B C$ in patients aged 61 and 63 years, respectively, treated by 

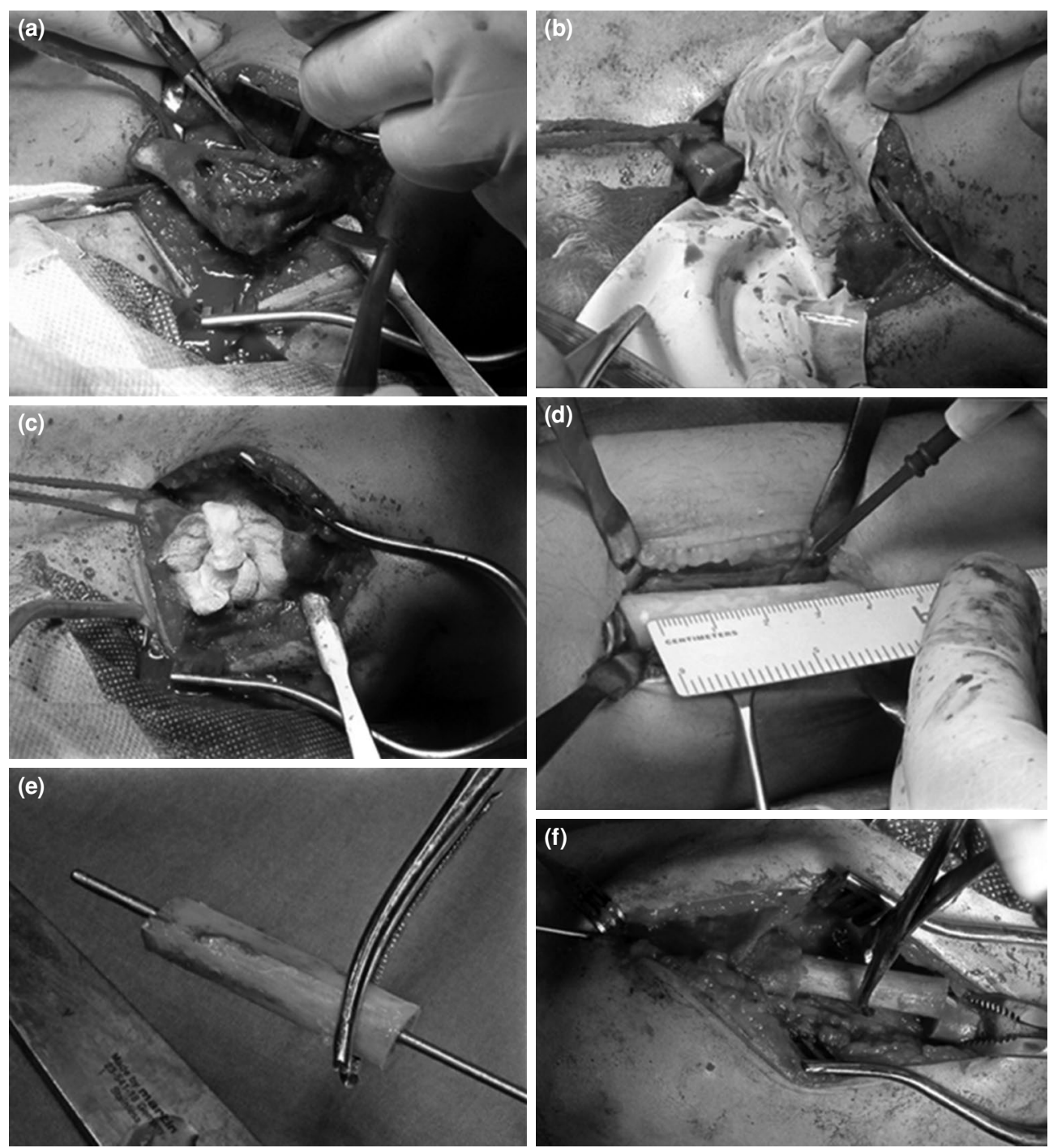

FIGURE 2. Intraoperative images. (a) Surgical exposure of aneurysmal bone cyst. (b) Resection. (c) Impregnation with lauromacrogol solution. (d) Subperiostal fibular graft measurement. (e) Placement of intramedullary titanium stem in autograft. (f) Retrograde-anterograde graft placement and stabilization under light compression.

en bloc resection without reconstruction of the bone defect. This option may be valid in older patients or patients with low functional demand, although we believe that it should not be considered in children or adolescents due to the potential secondary functional limitation.

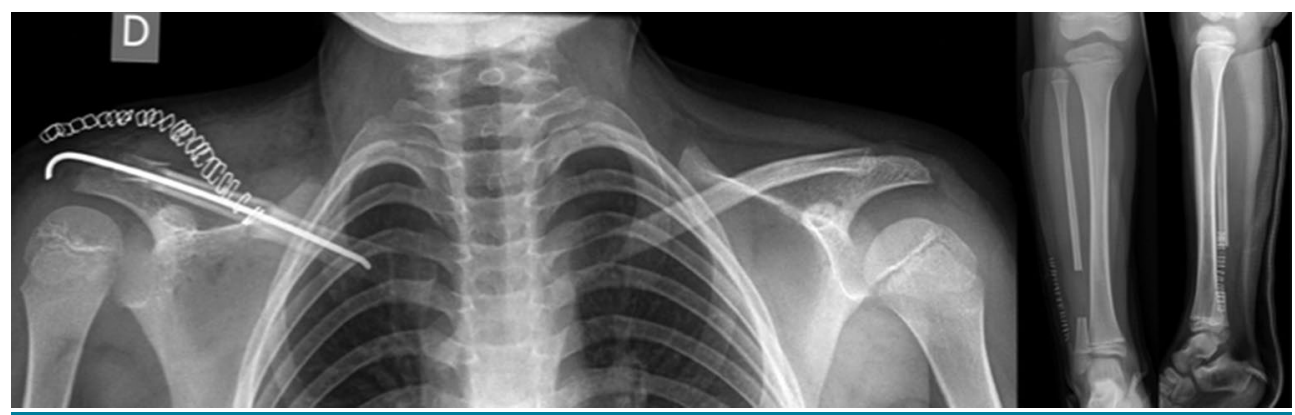

FIGURE 3. Immediate postoperative X-ray appearance. 


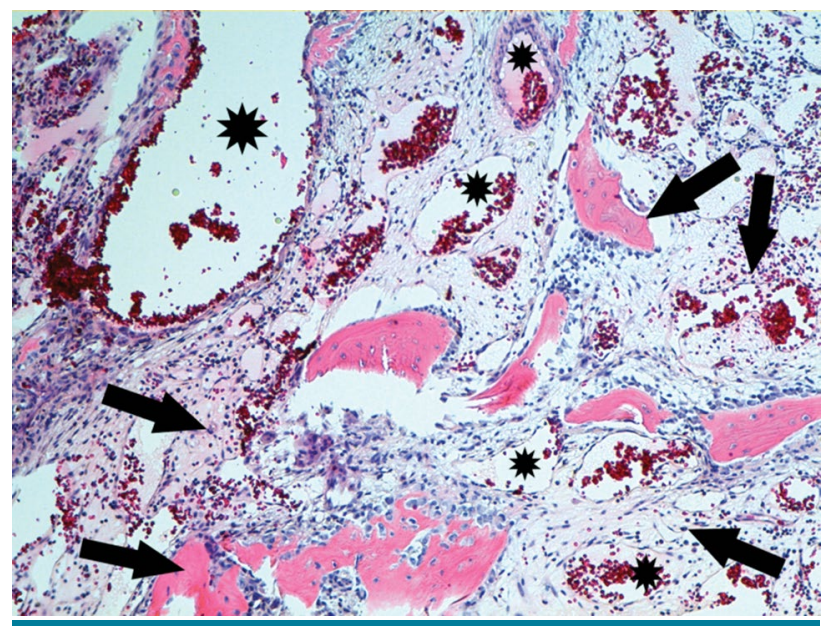

FIGURE 4. Aneurysmal bone cysts present large spaces filled with blood (black stars), separated by fibrovascular tissue with a degenerated calcifying fibromyxoid tissue, osteoid, bone, inflammatory, and giant cells (black arrows) $(\mathrm{H}-\mathrm{E} \times 10)$.
Chadha and Singh ${ }^{[10]}$ reported the case of a 26-yearold female patient with a large clavicle bone cyst treated by total claviculectomy, without complications or recurrence after two years of follow-up.

Curettage and graft have higher recurrence rates than resection and reconstruction, as shown by Kaiser $^{[4]}$ in his review of 13 cases of $A B C$ in the clavicle, 11 of which were treated by curettage with or without graft and one was treated by resection and reconstruction, without providing information about the treatment of one of the patients. In this series, there were nine recurrences in seven patients treated by curettage, without any recurrences in the patient treated by resection and reconstruction.

Heidt et al. ${ }^{[11]}$ reported a case of a successful clavicle reconstruction using a free vascularized fibula graft in a 10-year-old child, after a wide en-block resection resulting in a bone defect of $11 \mathrm{~cm}$ in length due to a total acromion-clavicular joint destruction. The initial
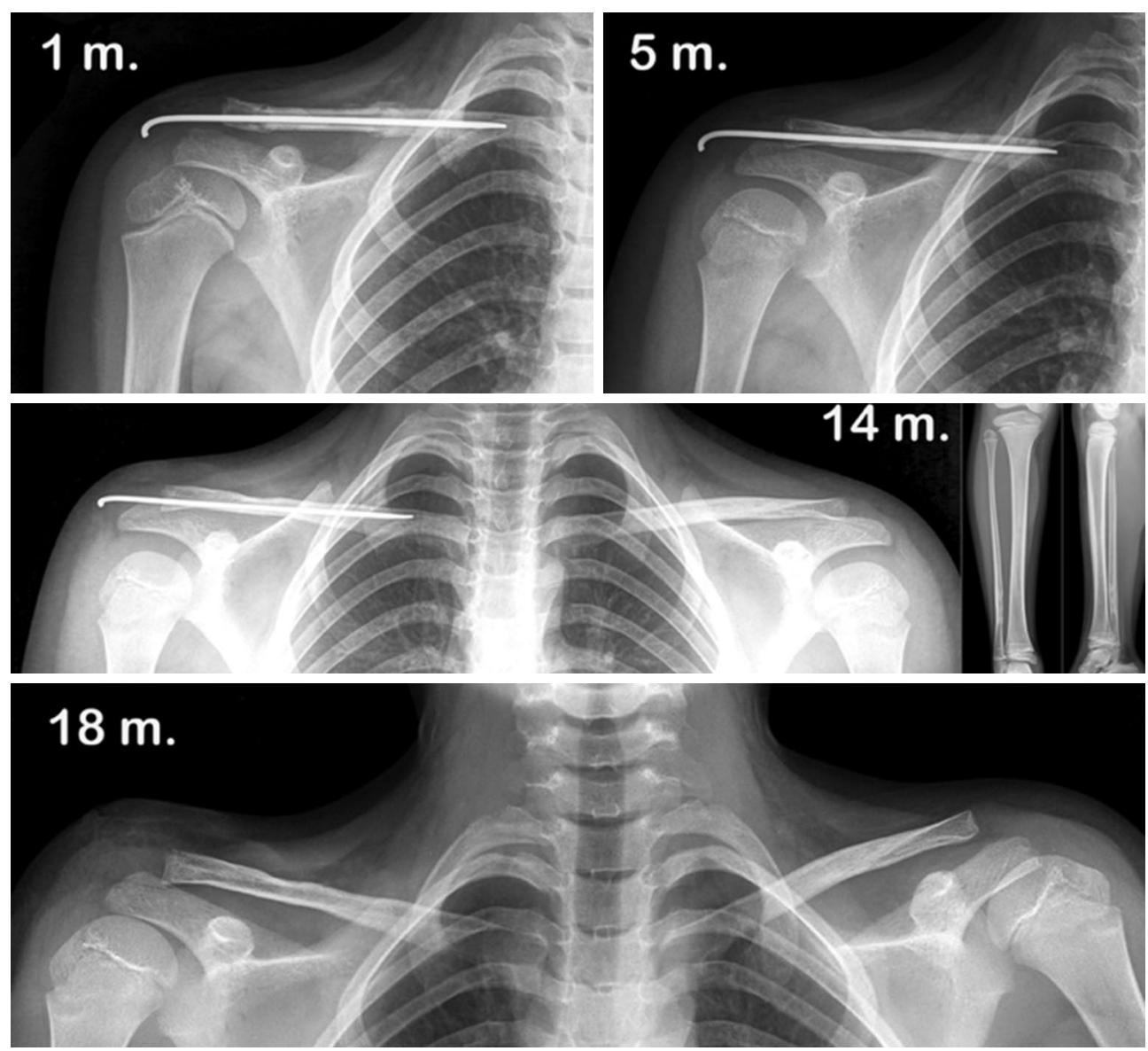

FIGURE 5. Postoperative follow-up. $(1 \mathrm{~m})$ : One month postoperatively. $(5 \mathrm{~m})$ : Five months postoperatively. (14 m): Fourteen months postoperatively, observe fibular reconstruction; (18 $\mathbf{~ m ) : ~}$ Eighteen months postoperatively. 
fixation with a 2.4 titanium elastic nail resulted in dislocation of the graft due to instability; therefore, an external fixator was applied with excellent result after four years of follow-up.

Arterial embolization is a complementary technique reserved for large sized $\mathrm{ABCs}$ or those located in highly vascularized areas, where invasive surgery might cause significant blood loss. ${ }^{[7,10]}$

Intralesional surgery or non-invasive methods such as intralesional injections were not considered given the size and location of the $A B C$ and the thinness of its bone walls with areas of discontinuity. In our case, we preferred block resection due to the lower rate of recurrence of this technique. In addition, once the $\mathrm{ABC}$ was resected, we impregnated the edges of the clavicle and the resection bed with lauromacrogol solution (Etoxisclerol ${ }^{\circledR} 2 \%$ ) to reduce the risk of possible recurrence. Finally, we performed a reconstruction of the clavicle by means of an autologous fibular bone graft, with the aim of achieving the best and quicker possible functional result. To avoid sequels in the donor area, we took the fibular graft subperiosteally, suturing the resulting periosteal sleeve, thus achieving a complete regeneration of the same, without clinical or functional consequences in said area. The stabilization of the graft by means of a $2 \mathrm{~mm}$ intramedullary titanium nail allowed a short period of immobilization of the child's arm, with a subsequent rapid functional recovery. Intralesional surgery or non-invasive methods such as intralesional injections would have conditioned a more limiting and prolonged postoperative physical activity for the child.

In our experience, bloc resection procedure and reconstruction by autologous fibular intercalary graft is a suitable therapeutic option for an ABC located in the clavicle diaphysis when its epiphyses may be preserved. If epiphysis cannot be preserved, we suggest alternative possible reconstruction methods as proposed by Heidt et al. ${ }^{[11]}$ rather a complete resection without reconstruction. In addition, we propose that the use of lauromacrogol topical solution may contribute to reducing the risk of recurrence, although this fact must be confirmed in larger series of patients. Furthermore, subperiosteal fibular graft obtaining favors the complete regeneration of bone in the donor area, without any consequent morbidity.

\section{Acknowledgments}

Special thanks to Dr. A. Sobrino-Prados from Servicio de Anatomía Patológica, Hospital Universitario Miguel Servet, for providing us the histological image of Figure 4.

\section{Declaration of conflicting interests}

The authors declared no conflicts of interest with respect to the authorship and/or publication of this article.

\section{Funding}

The authors received no financial support for the research and/or authorship of this article.

\section{REFERENCES}

1. Priemel MH, Stiel N, Zustin J, Luebke AM, Schlickewei C, Spiro AS. Bone tumours of the clavicle: Histopathological, anatomical and epidemiological analysis of 113 cases. J Bone Oncol 2019;16:100229

2. Zehetgruber H, Bittner B, Gruber D, Krepler P, Trieb K, Kotz $\mathrm{R}$, et al. Prevalence of aneurysmal and solitary bone cysts in young patients. Clin Orthop Relat Res 2005;439:136-43.

3. Mascard E, Gomez-Brouchet A, Lambot K. Bone cysts: unicameral and aneurysmal bone cyst. Orthop Traumatol Surg Res 2015;101(1 Suppl):S119-27.

4. Kaiser CL, Yeung CM, Raskin KA, Lozano-Calderon SA. Aneurysmal bone cyst of the clavicle: a series of 13 cases. J Shoulder Elbow Surg 2019;28:71-6.

5. Boubbou M, Atarraf K, Chater L, Afifi A, Tizniti S. Aneurysmal bone cyst primary--about eight pediatric cases: radiological aspects and review of the literature. Pan Afr Med J 2013;15:111.

6. Atik OŞ. Is there something new and interesting in my article? Eklem Hastalik Cerrahisi 2019;30:69.

7. Cottalorda J, Bourelle S. Modern concepts of primary aneurysmal bone cyst. Arch Orthop Trauma Surg 2007;127:105-14.

8. Yashavntha KC, Nalini KB, Menon J, Patro DK. Aneurysmal bone cyst of medial end of clavicle in a child, a rare case report. Indian J Surg Oncol 2014;5:158-60.

9. Smith J. Aneurysmal bone cyst of clavicle. Br J Radiol 1977;50:706-9.

10. Chadha M, Singh AP. Aneurysmal bone cyst of the clavicle. Can J Surg 2008;51:E32-3.

11. Heidt C, Ziebarth K, Erni D, Slongo T, Joeris A. Four years follow-up after clavicle reconstruction in a child: a case report. J Plast Reconstr Aesthet Surg 2014;67:1735-9. 